\title{
Phase Segregation of Polyester Based-Polyurethanes
}

\author{
Chi-Chin Chang, Kun-San Chen, T. Leon Yu, ${ }^{\dagger}$ \\ Yung-Sin Chen, Chin-Lung TsaI, and Yu-Hsien TsenG* \\ Department of Chemical Engineering, Yuan-Ze University, \\ Taoyuan, Nei-Li 32026, Taiwan \\ * Chemical Fiber Plant, Far Eastern Textile Co., Ltd. \\ Hsinpu, Hsinchu 30509, Taiwan
}

(Received March 17, 1999)

\begin{abstract}
Polyester based polyurethanes were synthesized from 4,4'-methylene bis(phenyl isocyanate) (MDI) with 1,4-butanediol as a chain extender and low molecular weight polyester-diol as a soft segment. Four polyesters were used in the synthesis of polyurethanes. Two of the polyesters were synthesized from monomers with even carbon atoms, i.e., adipic acid with 1,6-hexanediol (polyester-6-6) and adipic acid with 1,4-butanediol (polyester-6-4). The other two polyesters were synthesized from monomers with odd carbon atoms, i.e., pimelic acid with 1,5-pentanediol (polyester-7-5) and 1,5-pentanedioic acid with 1,5-pentanediol (polyester-5-5). The effects of compatibility between soft- and hard-segments and the chemical structures of polyesters on the phase segregation of soft- and hard-segments were studied by DSC and FT-IR.

KEY WORDS Polyurethane / Differential Scanning Calorimetry / Fourier Transform Infrared Spectroscopy / Phase Segregation /
\end{abstract}

The polyester based urethanes consist of an aromatic diisocyanate with a glycol chain extender as the hard segment and a low molecular weight polyester as the soft segment. They are considered to be linear segmented block copolymer, made up of alternating hard and soft block segments. It has been reported that soft- and hard-segmental phase segregation of polyurethanes is strongly controlled by the compatibility of these two segments and the chain mobility of hard segments inside the soft domain. ${ }^{1,2}$ Polyurethane of structure $-[(\mathrm{O}=$ C) $\left.\mathrm{NH}\left(\mathrm{CH}_{2}\right)_{n} \mathrm{NH}(\mathrm{C}=\mathrm{O}) \mathrm{O}\left(\mathrm{CH}_{2}\right)_{m} \mathrm{O}\right]$ - has been shown that when " $n$ " and " $m$ " are even the fit of each hydrogen donor group $(\mathrm{NH})$ to each electron donor group $(\mathrm{C}=\mathrm{O})$ should be relatively easy, whereas when " $n$ " or " $m$ " is odd the fit should not be perfect. ${ }^{3}$ Thus, in an irregular structure ( $n$ or $m$ is odd and fit is poor) not all groups should participate in hydrogen bonding, inter-molecular attractions should be weaker, and the melting point should be lower. It is known that the "zigzag" structure of polyester affects the geometric fit of polymer molecules and thus the crystallization behavior of polyesters.

In the present work, we studied the soft- and hardsegment phase segregation behavior of polyester basedpolyurethanes with two of the polyesters were synthesized from monomers with even carbon atoms, i.e., adipic acid with 1,6-hexanediol (polyeester-6-6) and adipic acid with 1,4-butanediol (polyester-6-4) and the other two polyesters were synthesized from monomers with odd carbon atoms, i.e., pimelic acid with 1,5-pentanediol (polyester-7-5) and 1,5-pentanoic diacid with 1,5pentanediol (polyester-5-5). Attempt has been made to correlate the polyurethane morphology with the polyester "zigzag" structures, the crystallinity of polyester soft segments, $\mathrm{NH}$ to $\mathrm{C}=\mathrm{O}$ structure geometric fit, and the compatibility between soft- and hardsegments. For polyurethanes with same polyester soft segmental molecular weights, with other things being

\footnotetext{
$\dagger$ To whom correspondence should be addressed.
}

equal, the soft- and hard-segmental compatibility increases with increasing polyester $-(\mathrm{C}=\mathrm{O})-\mathrm{O}$ - content. The polyesters consisting of even carbon or longer linear carbon monomers have a higher degree of crystallinity than those consisting of odd carbon or shorter linear carbon monomers. The mobility of urethane hard segments in the polyester soft domain with a lower degree of crystallinity (i.e., soft domain with higher free volume) should be higher than those in the polyester soft domain with a higher degree of crystallinity (i.e., soft domain with lower free volume). The effect of the mobility of urethane hard segments in the polyester soft domain on the morphology of polyurethane is discussed in this paper.

\section{EXPERIMENTAL}

\section{Polyester-Diol}

Four polyesters were synthesized using a conventional method. The chemical compositions of these polyesters are listed in Table I. The reaction temperature was increased by stepwise control as following: $140^{\circ} \mathrm{C} / 1 \mathrm{~h}$, $150^{\circ} \mathrm{C} / 1 \mathrm{~h}, 160^{\circ} \mathrm{C} / 1 \mathrm{~h}, 180^{\circ} \mathrm{C} / 2 \mathrm{~h}$, and $200^{\circ} \mathrm{C} / 4 \mathrm{~h}$. The acid and $-\mathrm{OH}$ values of the final polyesters determined by methods of ASTM D4262 and D4274 respectively are listed in Table I. The molecular weight distributions were determined by GPC (Waters model 746 with three Waters $\mu$-styragel columns which had molecular weight ranges of $0-10^{3}, 10^{2}-5 \times 10^{3}$, and $5 \times 10^{2}-2 \times 10^{4}$, and a RI detector) at $25^{\circ} \mathrm{C}$. Tetrahydrofuran (THF, Merck Co) was used as the mobile phase, and narrow MWD polystyrene standards (Aldrich Chemical $\mathrm{Co}$ ) were used in a linear calibration. The $\bar{M}_{n}$ and $\bar{M}_{w}$ of these polyesters are also listed in Table I.

\section{Polyurethane}

The polyester-diols synthesized from experimental procedure (a) were used to synthesize polyurethanes with diphenyl methane diisocyanate (MDI, Tokyo Kasei 
Table I. Chemical composition and molecular weight of polyester

\begin{tabular}{|c|c|c|c|c|}
\hline Sample & $\begin{array}{l}\text { Polyester- } \\
\quad 6-6\end{array}$ & $\begin{array}{c}\text { Polyester- } \\
7-5\end{array}$ & $\begin{array}{l}\text { Polyester- } \\
\text { 6-4 }\end{array}$ & $\begin{array}{l}\text { Polyester- } \\
5-5\end{array}$ \\
\hline Pimelic acid $/ \mathrm{mol}$ & - & 1.00 & - & - \\
\hline Adipic acid/mol & 1.00 & - & 1.00 & \\
\hline Pentanediolic acid $/ \mathrm{mol}$ & $\mathrm{l}-$ & - & - & 1.00 \\
\hline 1,6-Hexaneanediol $/ \mathrm{mo}$ & ol 1.20 & - & - & - \\
\hline 1,5-Pentanediol $/ \mathrm{mol}$ & - & 1.20 & - & - \\
\hline 1,4-Butanediol/mol & - & - & 1.19 & 1.19 \\
\hline $\begin{array}{l}\text { Acid value } / \mathrm{mg} \\
\mathrm{KOHg}^{-1}\end{array}$ & 3.81 & 3.60 & 3.91 & 3.88 \\
\hline $\begin{array}{l}\mathrm{OH} \text { value } / \mathrm{mg} \\
\mathrm{KOH} \mathrm{g}^{-1}\end{array}$ & 42.1 & 40.5 & 42.9 & 42.7 \\
\hline$M_{n}$ & 2660 & 2770 & 2443 & 2454 \\
\hline$M_{w} / M_{n}$ & 1.81 & 1.84 & 1.70 & 1.68 \\
\hline
\end{tabular}

Table II. Chemical compositions of polyurethanes

\begin{tabular}{|c|c|c|c|c|}
\hline \multirow{2}{*}{ Polyurethane $^{a}$} & Polyester & MDI & Butanediol & $\begin{array}{l}\text { Soft segment } \\
\text { content }\end{array}$ \\
\hline & $\mathrm{mol}$ & mol & mol & $w t \%$ \\
\hline PU-6-6 & 0.13 & 1.05 & 0.87 & 50.4 \\
\hline PU-7-5 & 0.13 & 1.05 & 0.87 & 51.4 \\
\hline PU-6-4 & 0.14 & 1.05 & 0.86 & 50.8 \\
\hline PU-5-5 & 0.14 & 1.05 & 0.86 & 50.9 \\
\hline
\end{tabular}

a The polyurethanes were synthesized from polyesters which has the same designated numbers as shown in Table I.

Kogyo Co.) and 1,4- butanediol (Riedel-de Haen Co.) as a chain extender in $N, N$-dimethyl formamide (DMF, Merck Co.) solvent by prepolymerization method. The chemical composition of these polyurethanes are shown in Table II. Two of these polyurethanes had polyester soft segments consisting of even number carbon monomers whilst the other two polyurethanes had polyester soft segments consisting of odd carbon atom monomers. Polyester-diol first reacted with diisocyanate at $90-100^{\circ} \mathrm{C}$ for one hour in DMF, (Merck Co.). The prepolymer then reacted with 1,4-butanediol at $90-100^{\circ} \mathrm{C}$ for another hour in DMF. The final polymer was then precipitated from methanol and the residual solvent was removed under vacuum at $60^{\circ} \mathrm{C}$ for $24 \mathrm{~h}$. The polymer was then compression molded on a press at $180^{\circ} \mathrm{C}$ for $10 \mathrm{~min}$ followed by cooling at an ambient temperature and environment. The samples were then kept at room temperature for at least one week before DSC and FT-IR measurements. Before polymerization, polyester was dried at $90^{\circ} \mathrm{C}$ under vacuum for $2 \mathrm{~h}$ to remove moisture and 1,4-butanediol and DMF were treated with molecular sieve (Merck, pore size $0.4 \mathrm{~nm}$ ) drying agent. MDI was used as it received without further purification. The final polyurethane contained $\sim 51.0$ $\mathrm{wt} \%$ of polyester.

\section{Differential Scanning Calorimetry (DSC)}

DSC measurements were carried out on a Du Pont 910 DSC. The heating rate was $10^{\circ} \mathrm{C} \mathrm{min}^{-1}$ for the temperature range of $-100-240^{\circ} \mathrm{C}$. The samples sizes were around $10 \mathrm{mg}$ for all measurements. Temperature calibration was done with a multiple indium-lead-nickel standard, an indium standard was used for heat flow calibration.
Table III. DSC data of polyesters

\begin{tabular}{lcccc}
\hline \multicolumn{1}{c}{ Polyester } & Polyester-6-6 & Polyester-7-5 & Polyester-6-4 & Polyeseter-5-5 \\
\hline$T_{m}(\mathrm{I}$-peak $) /{ }^{\circ} \mathrm{C}$ & 26.2 & 28.8 & - & 13.7 \\
$\Delta H(\mathrm{I}) / \mathrm{J} \mathrm{g}^{-1}$ & 4.8 & 8.9 & - & 66.1 \\
$T_{m}(\mathrm{II}-$ peak $) /{ }^{\circ} \mathrm{C}$ & 56.9 & 46.4 & 45.9 & - \\
$\Delta H(\mathrm{II}) / \mathrm{J} \mathrm{g}^{-1}$ & 107.4 & 92.5 & 80.3 & - \\
\hline
\end{tabular}

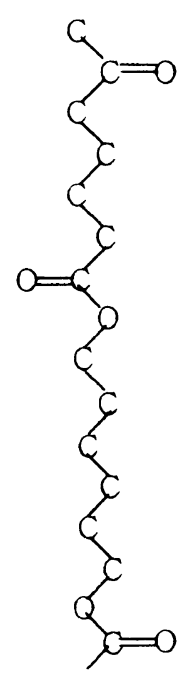

Polyester-6-6

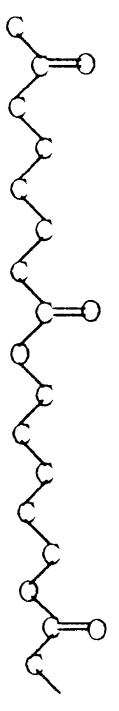

Polyester-7-5
Figure 1. Molecular conformations of polyester-7-5 and polyester-6-

Fourier Transform Infrared Spectroscopy (FT-IR)

A FT-IR Spectrometer (Perkin Elmer 1725X) was used to investigate hard segment $\mathrm{H}$-bond formation.

\section{RESULTS AND DISCUSSION}

\section{DSC Study}

DSC thermal analysis data of polyester-7-5, polyester6-6, polyester-5-5, and polyester-6-4 are summarized in Table III. The uncertainty with each temperature is approximately $\pm 1{ }^{\circ} \mathrm{C}$. Comparing the DSC data of polyester-7-5 with polyester-6-6 and of polyester-5-5 with polyester-6-4, it is obvious that for polyesters consisting of monomers with same number of carbon atoms, those consisting even number of carbon atoms have a higher melting temperature $\left(T_{\mathrm{m}}\right)$ and a higher enthalpy of fusion $(\Delta H)$ than those consisting of odd number of carbon atoms. That is $T_{\mathrm{m}}$ and $\Delta H$ of polyester-6-6 and polyester-6-4 are higher than those of polyester-7-5 and polyester-5-5 respectively. Since these four polyesters have similar molecular weight distribution, the differences of the crystallization behavior between polyester-6- 6 with polyester-7-5 and between polyester-6-4 with polyester5-5 could be due to different chemical structural conformations. As shown in Figure 1, polyester-6-6 has its $\mathrm{C}=\mathrm{O}$ double bond in syndiotactic conformation whilst polyester-7-5 has its $\mathrm{C}=\mathrm{O}$ bonds in isotactic conformation. Similarly, polyester- $6-4$ and polyester-5-5 have their $\mathrm{C}=\mathrm{O}$ double bond in syndio- and iso-tactic conformations respectively. The "zigzag" structure has a strong effect on the crystallization behavior of 
polyesters. The DSC results also show that polyesters containing monomers with longer carbon atoms (i.e., polyesters-6-6 and -7-5) have higher melting temperature and heat of fusion than those containing monomers with short carbon atoms (i.e., polyesters-6-4 and -5-5). These DSC results suggest that the tendency of polyester crystallization decreases sequentially as following: polyester- $6-6>$ polyester-7-5 $>$ polyester-6-4 $>$ polyester-5-5.

DSC curves of polyurethanes annealed at room temperature for one week are shown in Figure 2. The positions of the DSC thermal transition, endothermic peak temperatures and enthalpies of fusion are listed in Table IV. DSC thermal behavior is apparent in five temperature ranges. The soft segment glass transition temperature $\left(T_{\mathrm{g} 1}\right)$ appears at $-33,-44,-49$, and $-40^{\circ} \mathrm{C}$ for PU-6-6, PU-7-5, PU-6-4, and PU-5-5 respectively. $T_{\mathrm{g} 1}$ values can be used as an indication of relative purity of the soft segment regions. The more soft segment domains are contaminated with the dissolved high glass transition temperature hard segments, the higher soft domain $T_{\mathrm{g} 1}$. The extent of hard segment/ soft segment mixing will depend on the overall hard segment content, both segment lengths, and the affinity of one segment for the other. The lower soft segment $T_{\mathrm{g} 1}$ indicates that PU has a higher degree of soft- and hard-segment phase segregation. ${ }^{4}$ When there are hard segments dispersed in the soft domains, the $T_{\mathrm{g} 1}$ is raised.

The second region of interest is that corresponding to the melting of soft segment $\left(T_{\mathrm{m} 1}\right.$, around $\left.11.0-56^{\circ} \mathrm{C}\right)$. Comparing Table III and Table IV, it is obvious that polymerization of polyesterdiol with MDI and 1,4butanediol leads to decreases both in the melting temperature $T_{\mathrm{m} 1}$ and melting enthalpy $\Delta H_{1}$ of polyester soft segments. The $T_{\mathrm{m} 1}$ and $\Delta H_{1}$ decreased sequentially as following: PU-6-6 > PU-6-4 > PU-7-5 > PU-5-5. $T_{\mathrm{m} 1}$ and $\Delta H_{1}$ of soft segments are indicators of soft segments crystallization. ${ }^{4}$ The experimental results revealed that the ordering of soft segment domain decreased as following: PU-6-6 > PU-6-4 > PU-7-5 > PU-5-5.

The remaining regions of DSC thermal behavior result from endotherms associated with hard segment domains. The endotherm around $52-88^{\circ} \mathrm{C}\left(T_{\mathrm{m} 2}(\mathrm{I})\right)$ results from the breakup of hard segments with short range ordering domain induced by room temperature annealing. ${ }^{5}$ The endotherm around $120-175^{\circ} \mathrm{C}\left(T_{\mathrm{m} 2}\right.$ (II)) corresponds to the melting of hard segment domain with long range ordering. The endotherm around $175-220^{\circ} \mathrm{C}\left(T_{\mathrm{m} 2}\right.$ (III)) corresponds to the melting of microcrystalline hard segment. From DSC results, we found that PU-6-4, PU-7-5 and PU-5-5 had microcrystalline hard segment domains with $T_{\mathrm{m} 2}$ (III) and $\Delta H_{\mathrm{m} 2}$ (III) decreasing sequentially. The DSC data of PU-7-5, PU-5-5, and PU-6-6 showed small endotherm-II indicating the presence of long range ordering hard segment domains. The $T_{\mathrm{m} 2}$ (II) of these polyurethanes decreased sequentially as following: PU-7-5> PU-5-5>PU-6-6. In summary of DSC results, we might conclude that degree of hard segment ordering decreased as following: PU-6-4 > PU-7-5 > PU-5-5 > PU-6-6. It is noticed that the $T_{\mathrm{g} 1}$ of these polyurethanes increased in the contrary sequence, i.e., PU-6-4 < PU-7-5 < PU-5-5 < PU-6-6.

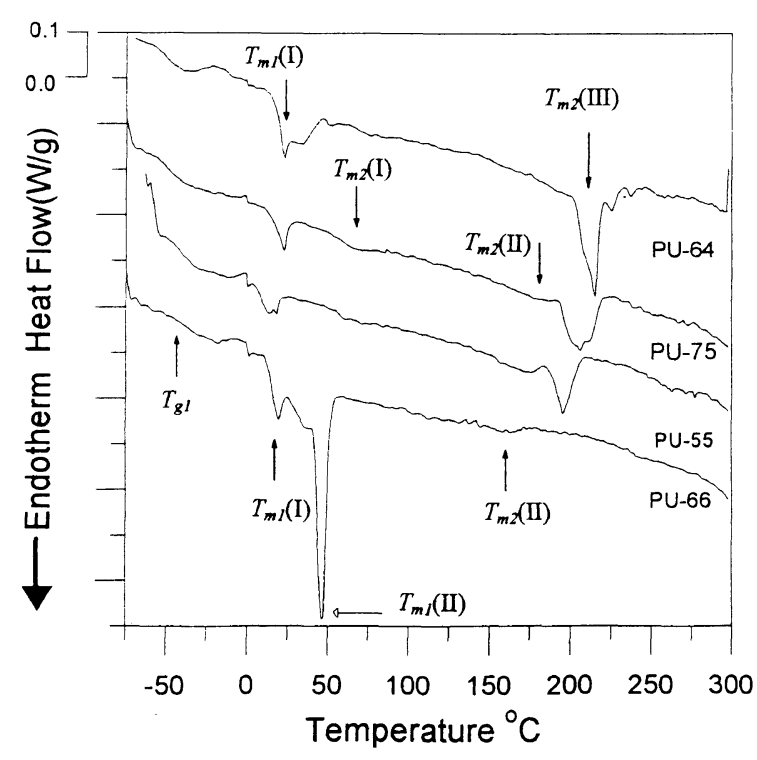

Figure 2. DSC curves of polyurethanes.

The higher $T_{\mathrm{m} 2}$ of PU indicates a higher degree of ordering of hard segment domain, whilst the lower $T_{\mathrm{g} 1}$ indicates a higher degree of soft and hard segmental phase segregation. Increasing the ordering of hard segmental domain would cause an increase in the soft and hard segmental phase separation. Thus the $T_{\mathrm{m} 2}$ and $T_{\mathrm{g} 1}$ data of these polyurethanes were in agreement with each other and the degree of soft and hard segmental phase segregation decreased sequentially as following: PU-6-4, PU-7-5, PU-5-5, and PU-6-6.

\section{FT-IR Study}

Almost all of the infrared research on PU has been focused on two principal vibrational regions: the $\mathrm{N}-\mathrm{H}$ stretching vibration $\left(3200-3500 \mathrm{~cm}^{-1}\right)$, and the $\mathrm{C}=\mathrm{O}$ stretching vibration amide-I region $(1637-1730$ $\left.\mathrm{cm}^{-1}\right){ }^{6-13}$ In this investigation, FT-IR was used to study the effect of polyester structure on the hydrogen bonding behavior of PU. The room temperature infrared spectra for PU-6-4, PU-7-5, PU-5-5, and PU-6-6 annealed at room temperature are shown in Figure 3. There are distinct differences between the spectra at $\mathrm{N}-\mathrm{H}$ stretching and $\mathrm{C}=\mathrm{O}$ stretching regions with different polyester structures, mainly due to peak shifts and intensity changes.

It is well-known that the infrared absorbance of $\mathrm{H}$-bonded urethane carbonyl appeared at lower wave numbers than that of free urethane carbonyl. ${ }^{9-11}$ The amide-I infrared spectra of semi-crystalline samples such as nylons ${ }^{12}$ and polyurethanes ${ }^{13}$ have been reported to comprise distinct spectral features due to the degree of carbonyl $\mathrm{H}$-bonding. In order of increasing wave numbers, these are $\mathrm{H}$-bonded carbonyl groups in ordered ("crystalline") soft domains (1624-1656 $\left.\mathrm{cm}^{-1}\right), \mathrm{H}$ bonded carbonyl groups in ordered ("crystalline") hard domains (1699-1706 $\mathrm{cm}^{-1}$ ), H-bonded carbonyl groups in disordered ("amorphous") conformations (1714$1718 \mathrm{~cm}^{-1}$ ), and non-H-bonded (free) carbonyl groups $\left(1731-1733 \mathrm{~cm}^{-1}\right)$. In polyester based polyurethanes, most of the free carbonyl groups are contributed from polyester $\mathrm{C}=\mathrm{O}$ group. Similarly, two distinct bands are 


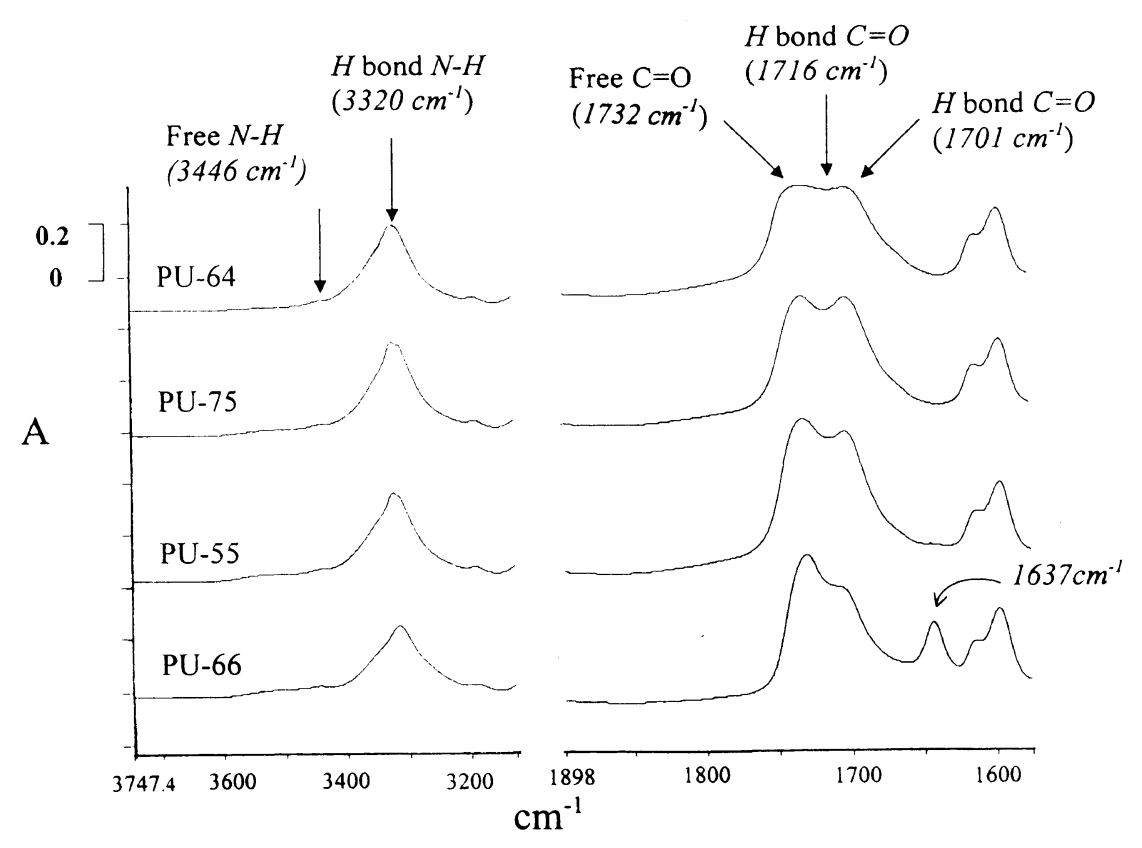

Figure 3. FT-IR spectra of polyurethanes.

observed in $\mathrm{N}-\mathrm{H}$ stretching region, i.e., $3320-3329$ and $3441-3446 \mathrm{~cm}^{-1}$ which are attributed to H-bonded and "free" N-H stretching vibrations respectively. ${ }^{14}$

As shown in Figure 3, the IR spectrum of PU-6-6 exhibits three $\mathrm{C}=\mathrm{O}$ amide-I bands, i.e., strong free carbonyl stretching $\left(1732 \mathrm{~cm}^{-1}\right)$, weak shoulder $\mathrm{H}$ bonded carbonyl stretching in hard domains (1701 $\mathrm{cm}^{-1}$ ), and a medium $\mathrm{H}$-bonded carbonyl stretching in ordered soft domains $\left(1637 \mathrm{~cm}^{-1}\right)$. These results suggested very weak $\mathrm{H}$-bond formed in the hard segments. The appearance of $\mathrm{H}$-bonded carbonyl stretching at $1637 \mathrm{~cm}^{-1}$ indicating the dispersion of hard segments in the ordered soft domain which caused the formation of $\mathrm{H}$-bond between urethane $-\mathrm{NH}$ and ester $\mathrm{C}=\mathrm{O}$. Skrovanek et al. ${ }^{12}$ using FT-IR, studied H-bond formation of nylon 11. Their results showed three amide-I carbonyl stretching bands, i.e., free carbonyl groups $\left(1680 \mathrm{~cm}^{-1}\right), \mathrm{H}$-bonded carbonyl groups in disordered conformation ("amorphous") $\left(1656 \mathrm{~cm}^{-1}\right)$, and $\mathrm{H}$ bonded carbonyl groups in ordered conformation ("crystalline") $\left(1638 \mathrm{~cm}^{-1}\right)$. The interesting thing is that the peak frequency of $\mathrm{H}$-bonded carbonyl stretching in ordered domains $\left(1638 \mathrm{~cm}^{-1}\right)$ of nylon 11 is very close to that of H-bonded carbonyl in soft domain of PU-6-6. Since nylon 11 is an aliphatic polyamide, so we believe that $\mathrm{H}$-bonded urethane $\mathrm{NHC}=\mathrm{O}$ group dispersed in polyester ordered domain might contribute to $\mathrm{C}=\mathrm{O}$ stretching at $1637 \mathrm{~cm}^{-1}$ of PU-6-6.

Three $\mathrm{C}=\mathrm{O}$ amide-I stretching bands were observed in IR spectrum of PU-6-4, i.e., strong H-bonded carbonyl stretching in ordered hard domains $\left(1701 \mathrm{~cm}^{-1}\right)$, medium $\mathrm{H}$-bonded carbonyl stretching disordered hard domains $\left(1716 \mathrm{~cm}^{-1}\right)$, and free carbonyl groups $\left(1732 \mathrm{~cm}^{-1}\right)$. The $\mathrm{C}=\mathrm{O}$ stretching at $1716 \mathrm{~cm}^{-1}$ could be due to the $\mathrm{H}$-bonded ester $\mathrm{C}=\mathrm{O}$ groups which were covalent bonded near the hard segments and caused the formation of disordered hard segment domains. The ease of $-\mathrm{NH}$ to $\mathrm{C}=\mathrm{O}$ fit for $\mathrm{H}$-bonded $\mathrm{C}=\mathrm{O}$ stretching of $1716 \mathrm{~cm}^{-1}$ absorbance depends on the perfection of urethane $-\mathrm{NH}$

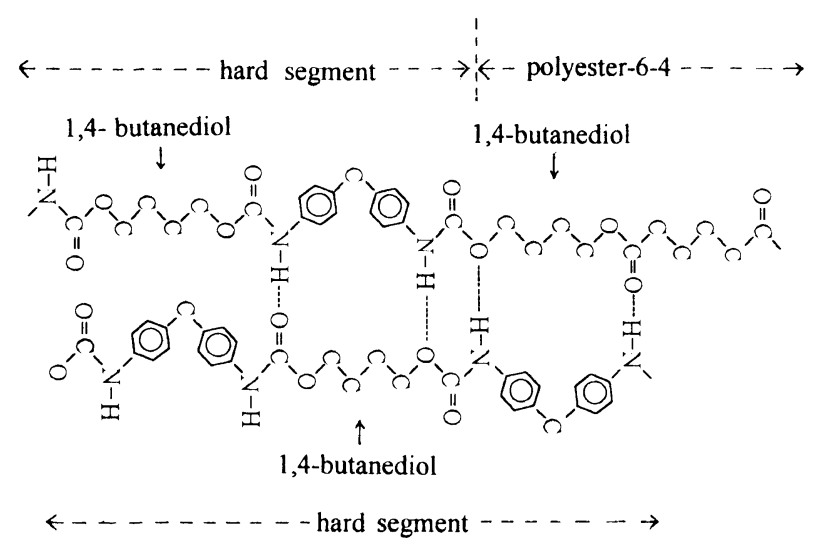

Figure 4. Geometric fit of hard segment urethane $-\mathrm{NH}$ to soft segment ester $\mathrm{C}=\mathrm{O}$ group which was covalent bonded near the hard segments of PU-6-4.

to ester $\mathrm{C}=\mathrm{O}$ geometric fit. The ester groups composed of similar chemical structure as that of hard segments should be easy to form $\mathrm{H}$-bond with urethane $-\mathrm{NH}$. As shown in Figure 4, PU-6-4 composed 1,4-butanediol monomer both in hard and polyester soft segments which led to a better urethane $-\mathrm{NH}$ to ester $\mathrm{C}=\mathrm{O}$ geometric fit than other polyurethanes. Thus only the IR spectrum of PU-6-4 shows the $\mathrm{C}=\mathrm{O}$ stretching at $1716 \mathrm{~cm}^{-1}$. These presence of strong $1701 \mathrm{~cm}^{-1}$ and $1716 \mathrm{~cm}^{-1}$ IR absorbance indicate a very strong $\mathrm{H}$-bond formation between $-\mathrm{NH}$ and $\mathrm{C}=\mathrm{O}$ groups of PU-6-4.

Two $\mathrm{C}=\mathrm{O}$ amide-I stretching bands were observed in IR spectra of PU-7-5 and PU-5-5, i.e., strong free carbonyl stretching $\left(1732 \mathrm{~cm}^{-1}\right)$ and strong H-bonded carbonyl stretching in hard domains $\left(1701 \mathrm{~cm}^{-1}\right)$. Because the polyester soft segment were synthesized from odd carbon monomers, the degree of soft segment crystallinity and the perfection urethane $-\mathrm{NH}$ to ester $\mathrm{C}=\mathrm{O}$ fit of PU-7-5 and PU-5-5 are not so good as that of PU-6-6 and PU-6-4 respectively. Neither H-bonded carbonyl stretching in ordered soft domain $\left(1637 \mathrm{~cm}^{-1}\right)$ 
nor H-bonded carbonyl stretching in disordered hard domains $\left(1716 \mathrm{~cm}^{-1}\right)$ was observed in the IR spectra of PU-7-5 and PU-5-5.

The H-bonded $\mathrm{N}-\mathrm{H}$ stretching frequency does not exhibit separate features attributable to ordered and disordered $\mathrm{H}$-bonded conformations but rather reflects the overall distribution of $\mathrm{H}$-bonded strength. In Figure 3 , the peak height of $1701 \mathrm{~cm}^{-1}$ absorbance band decreased and $\mathrm{N}-\mathrm{H}$ stretching vibration of $3446 \mathrm{~cm}^{-1}$ increased sequentially for PU-6-4, PU-7-5, PU-5-5, and PU-6-6. These results suggested that the degree of hard segmental $\mathrm{H}$-bonding decreased sequentially for PU-6-4, PU-7-5, PU-5-5, and PU-6-6.

\section{Discussion}

The compatibility between the soft- and hard-segments (compatibility enhances with the - $\mathrm{COO}$ - content of polyester soft segment), the degree of crystallinity of soft segments (the polyesters consist of longer linear carbon monomers or even carbon monomers have a higher degree of crystallinity), and the perfection of $-\mathrm{NH}$ to $-\mathrm{C}=\mathrm{O}$ fit among the urethane hard segments for $\mathrm{H}$-bond formation (monomers with even carbon atoms have a better fit than those with odd carbon atoms) are three key factors to control the degree of ordering of hard segments and the soft- and hard-segmental phase segregation. ${ }^{15,16}$

The polyester soft segments of PU-6-6 and PU-7-5 have similar molecular weight and similar number of carboxyl group per polyester molecular chain, hence the compatibility between soft and hard segments of these two polyurethanes should be similar. Similarly, PU-6-4 and PU-5-5 have similar soft and hard segmental compatibility. However, owing to the lower content of polyester COO- group per polymer chain, the soft and hard segmental compatibility of PU-6-6 and PU-7-5 should be poor than that of PU-6-4 and PU-5-5.

The polyesters consisting of even carbon or longer linear carbon monomers have a higher degree of crystallinity than those consisting of odd carbon or shorter linear carbon monomers. Since the polyester of PU-6-6 consisted of monomers with even carbon atoms and longer linear carbon chains (repeat unit of polyester-6-6 consisted of 12 carbon atoms), PU-6-6 had a highest degree of soft segment crystallinity among these polyurethanes ( $T_{\mathrm{m}}$ of polyester- $6-6$ was $56.9^{\circ} \mathrm{C}$ (Table III) and $T_{\mathrm{m} 1}$ of PU-6-6 was $46.4^{\circ} \mathrm{C}$ (Table IV)). The polyester of PU-7-5 consisted of monomers with odd carbon atoms and longer linear carbon chains (repeat unit of polyester-7-5 consisted of 12 carbon atoms) whilst the polyester of PU-6-4 consisted of monomers with even carbon atoms and shorter linear carbon chains (repeat unit of polyester-6-4 consisted of 10 carbon atoms), thus PU-7-5 and PU-6-4 had similar degree of soft segment crystallinity $\left(T_{\mathrm{m}}\right.$ of polyester-7-5 and polyester-6-4 were 46.4 and $45.9^{\circ} \mathrm{C}$ respectively (Table III) and $T_{\mathrm{m} 1}$ of PU-7-5 and PU-6-4 were 22.4 and $21.9^{\circ} \mathrm{C}$ respectively (Table IV)). However, the degrees of polyester crystallinity of PU-7-5 and PU-6-4 were lower than that of PU-6-6. The polyester of PU-5-5 consisted of monomers with odd carbon atoms and shorter linear carbon chains (repeat unit of polyester-5-5 consisted of 10 carbon atoms), thus PU-5-5 had the lowest degree of soft segment
Table IV. DSC data of polyurethanes annealed at room temperature

\begin{tabular}{lrrrr}
\hline \multicolumn{1}{c}{ Polyurethane } & PU-6-6 & PU-7-5 & PU-6-4 & PU-5-5 \\
\hline$T_{\mathrm{g} 1} /{ }^{\circ} \mathrm{C}$ & -33.3 & -44.4 & -48.6 & -40.2 \\
$T_{\mathrm{m} 1}(\mathrm{I}-$ peak $) /{ }^{\circ} \mathrm{C}$ & 18.7 & 22.4 & 21.9 & 13.0 \\
$T_{\mathrm{m} 1}(\mathrm{II}-$ peak $) /{ }^{\circ} \mathrm{C}$ & 46.4 & & & \\
$\Delta H_{\mathrm{m} 1}(\mathrm{I}+\mathrm{II}) / \mathrm{J} \mathrm{g}^{-1}$ & 34.8 & 4.2 & 11.4 & 5.2 \\
$T_{\mathrm{m} 2}(\mathrm{I}-$ peak $) /{ }^{\circ} \mathrm{C}$ & & 66.1 & 76.0 & 61.0 \\
$\Delta H_{\mathrm{m} 2}(\mathrm{I}) / \mathrm{J} \mathrm{g}{ }^{-1}$ & & 3.2 & 0.9 & 1.6 \\
$T_{\mathrm{m} 2}(\mathrm{II}-\mathrm{peak}) /{ }^{\circ} \mathrm{C}$ & 158.2 & 176.8 & & 174.8 \\
$\Delta H_{\mathrm{m} 2}(\mathrm{II}) / \mathrm{J} \mathrm{g}{ }^{-1}$ & 3.2 & & & \\
$T_{\mathrm{m} 2}(\mathrm{III}-\mathrm{peak}) /{ }^{\circ} \mathrm{C}$ & & 198.2 & 214.9 & 195.3 \\
$\Delta H_{2}(\mathrm{II}+\mathrm{III}) / \mathrm{J} \mathrm{g}^{-1}$ & & 19.4 & 27.0 & 16.3 \\
\hline
\end{tabular}

The subscripts 1 and 2 correspond to soft and hard segments respectively.

crystallinity $\left(T_{\mathrm{m}}\right.$ of polyester-5-5 was $13.7^{\circ} \mathrm{C}$ (Table III) and $T_{\mathrm{m} 1}$ of PU-5-5 was $13.0^{\circ} \mathrm{C}$ (Table IV)).

Since all of the hard segments of these polyurethanes consisted of MDI and 1,4-butanediol, thus the degree of the fit of $-\mathrm{NH}$ to $-\mathrm{C}=\mathrm{O}$ depended on the chemical structures of ester groups covalent bonded near urethane hard segments. It is known that ester repeat units consisting of even carbon monomers have better geometric fit than those consisting of odd carbon monomers. The urethanes covalent bonded to polyesters consisting of even carbon monomers should have a better $-\mathrm{NH}$ to $-\mathrm{C}=\mathrm{O}$ fit than those bonded to polyesters consisting of odd carbon monomers. Thus PU-6-6 and PU-6-4 had a better $-\mathrm{NH}$ to $\mathrm{C}=\mathrm{O}$ fit than PU-7-5 and PU-5-5. In contrast to other polyurethanes, both the soft and hard segments of PU-6-4 contained 1,4-butanediol monomer, thus we might conclude that PU-6-4 had a best urethane $-\mathrm{NH}$ to $-\mathrm{C}=\mathrm{O}$ fit in these polyurethanes.

After finishing polymerization and molding the polyurethanes at high temperatures (i.e., $180^{\circ} \mathrm{C}$ in this work), some of the hard segments might be mixed into the polyester soft segments. Once the polyurethanes are cooled from a high temperature to room temperature, the hard segments with a higher mobility are easy to move out from the soft domain and aggregate to form $\mathrm{H}$-bond. The mobility of urethane hard segments in the polyester soft domain with a lower degree of crystallinity (i.e., soft domain with higher free volume) should be higher than those in the polyester soft domain with a higher degree of crystallinity (i.e., soft domain with lower free volume). Since PU-6-6 had a highest degree of soft segment crystallinity, the viscosity of PU-6-6 was much higher than that of other polyurethanes. Thus the mobility of hard segments inside the soft domain of PU-6-6 was much lower than that of other polyurethanes while the polyurethanes were cooled from a high temperature to room temperature. Thus the urethane hard segments were fixed and dispersed inside the soft domain and very few hard segment aggregation through was observed. No $T_{\mathrm{m} 2}$ (III) endotherm was observed in DSC data of PU-6-6 (Figure 2 and Table IV). The FT-IR spectrum of PU-6-6 shows a weak shoulder H-bond $-\mathrm{C}=\mathrm{O}$ stretching in hard domains $\left(1701 \mathrm{~cm}^{-1}\right)$ and a medium $\mathrm{H}$-bonded carbonyl stretching in ordered soft domains $\left(1637 \mathrm{~cm}^{-1}\right)$ indicating the dispersion of hard segments inside the ordered soft domains and very few ordered hard segment domains occurred. 
The degrees of crystallinity of polyester-7-5 and polyester-6-4 were similar and lower than that of polyester-6-6. Thus the mobility of hard segments of PU-7-5 and PU-6-4 was lower than that of PU-6-6. Though the compatibility between soft and hard segments of PU-6-4 was higher than that of PU-7-5, however, the $-\mathrm{NH}$ to $-\mathrm{C}=\mathrm{O}$ fit of PU-6-4 was much better than that of PU-7-5, thus PU-6-4 had a higher degree of $-\mathrm{NH}$ to $-\mathrm{C}=\mathrm{O} \mathrm{H}-$ bond formation than PU-7-5. The DSC data showed that PU-6-4 had highest values of $T_{\mathrm{m} 2}$ (III) and $\Delta \mathrm{H}_{2}$ (II + III) and PU-7-5 had the next higher values in all of these polyurethanes. The FT-IR spectrum of PU-6-4 shows a strong $\mathrm{H}$-bonded $\mathrm{C}=\mathrm{O}$ stretching at $1701 \mathrm{~cm}^{-1}$ and a medium $\mathrm{H}$-bonded $\mathrm{C}=\mathrm{O}$ stretching at $1716 \mathrm{~cm}^{-1}$ indicating very strong $-\mathrm{NH}$ to $\mathrm{C}=\mathrm{O} \mathrm{H}$-bond formation in ordered and disordered hard domains. However, no $\mathrm{H}$-bonded $\mathrm{C}=\mathrm{O}$ stretching at $1716 \mathrm{~cm}^{-1}$ was observed in the IR spectrum of PU-7-5.

The polyester soft segments of PU-5-5 had the lowest degree of crystallinity in all of these polyurethanes. Thus PU-5-5 had the highest hard segment mobility inside the soft domain. However, owing to the higher compatibility between soft and hard segments (because of higher-COO content in polyester soft segments) and poor $-\mathrm{NH}$ to $\mathrm{C}=\mathrm{O}$ geometric fit, $\mathrm{PU}-5-5$ had a degree of hard segment crystallinity higher than PU-6-6 and lower than PU-7-5. As shown in DSC data, PU-5-5 had $T_{\mathrm{m} 2}$ and $\Delta \mathrm{H}_{2}(\mathrm{II}+\mathrm{III})$ values lower than PU-6-4 and PU-7-5 but higher than PU-6-6. Similarly, IR spectra showed that PU-5-5 had a lower $1701 \mathrm{~cm}^{-1} \mathrm{H}$-bonded $\mathrm{C}=\mathrm{O}$ stretching than PU-6-4 and PU-7-5 but higher than PU-6-6.

\section{CONCLUSION}

In the present study, we show that the soft and hard segmental phase segregation of polyester based polyurethanes strongly depends on: (1) the mobility of hard segments inside the soft domains, (2) the compatibility between soft and hard segments and (3) the $-\mathrm{NH}$ to $\mathrm{C}=\mathrm{O}$ geometric fit for $\mathrm{H}$-bond formation. The polyesters composed of monomers with even and long carbon atoms have a high degree of crystallinity which reduced the mobility of hard segments. The even carbon monomers of polyester segments also improve the $-\mathrm{NH}$ to $\mathrm{C}=\mathrm{O}$ geometric fit, especially for PU-6-4 in which both soft and hard segments contain same monomer, i.e., 1,4-butanediol. The morphology of polyurethanes was a balanced result of these factors.

Acknowledgment. The authors would like to thank Far Eastern Textile Co., Ltd. for financial support through grant CFF-87002.

\section{REFERENCES}

1. Y. Li, T. Gao, and B. Chu, Macromolecules, 25, 1737 (1992)

2. Y. Camberlin and J. P. Pascault, J. Polym. Sci., Polym. Phys. Ed., 22, 1835 (1984).

3. J. H. Saunders and K. C. Frisch, in "Polyurethanes Chemistry and Technology," Interscience Publishers, New York, N.Y., 1962, Part I, Chapter 6.

4. T. R. Hesketh, J. W. C. van Bogart, and S. L. Cooper, Polym. Eng. Sci., 20, 190 (1980).

5. R. W. Seymour and S. L. Cooper, Macromolecules, 6, 48 (1973).

6. J. W. C. Van Bogart, D. A. Bluemke, and S. L. Cooper, Polymer, 22, 1428 (1981)

7. D. J. Skrovanek, S. E. Howe, P. C. Painter, and M. M. Coleman, Macromolecules, 18, 1676 (1985)

8. F. Papadimtrakopoulos, E. Sawa, and W. J. MacKnight, Macromolecules, 25, 4682 (1992).

9. M. M. Coleman, K. H. Lee, D. J. Skrovanek, and P. C. Painter, Macromolecules, 19, 2149 (1986).

10. R. W. Seymour, G. M. Estes, and S. L. Cooper, Macromolecules, 3, 579 (1970).

11. W. J. MacKnight and W. J. Yang, J. Polym. Sci., Polym. Symp., 42, 817 (1973).

12. D. J. Skrovanek, P. C. Painter, and M. M. Coleman, Macromolecules, 19, 699 (1986).

13. S. K. Pollack, D. Y. Shen, S. L. Hsu, Q. Wang, and H. D. Stidham, Macromolecules, 22, 270 (1988).

14. F. C. Wang, M. Feve, T. M. Lam, and J. P. Pascault, J. Polym. Sci., Polym. Phys. Ed., 32, 1305 (1994).

15. S. L. Chang, T. L. Yu, C. C. Huang, W. C. Chen, K. Linliu, and T. L. Lin, Polymer, 39, 3479 (1998)

16. K. S. Chen, T. L. Yu, and Y. H. Tseng, J. Polym. Sci., Polym. Chem. Ed, in press. 